\title{
An Integral View of Balmer-dominated Shocks in Supernova Remnants
}

\author{
Sladjana Nikolić ${ }^{1}$, Glenn van de Ven ${ }^{1}$, Kevin Heng ${ }^{2}$, Daniel Kupko ${ }^{3}$, \\ Jose Alfonso Lopez Aguerri ${ }^{4}$, Jairo Méndez-Abreu ${ }^{4}$, Joan Font Serra ${ }^{4}$ \\ and John Beckman ${ }^{4}$ \\ ${ }^{1}$ Max Planck Institute for Astronomy, Königstuhl 17, Heidelberg, Germany \\ email: nikolic@mpia.de \\ ${ }^{2}$ University of Bern, Center for Space and Habitability, Sidlerstrasse 5, Bern, Switzerland \\ ${ }^{3}$ Leibniz Institute for Astrophysics Potsdam (AIP), An der Sternwarte 16, Potsdam, Germany \\ ${ }^{4}$ Instituto de Astrofísica de Canarias (IAC), Vía Láctea, La Laguna (Tenerife), Spain
}

\begin{abstract}
We present integral-field spectroscopic observations with the VIMOS-IFU at the VLT of fast (2000-3000 $\mathrm{kms}^{-1}$ ) Balmer-dominated shocks surrounding the northwestern rim of the remnant of supernova 1006. The high spatial and spectral resolution of the instrument enable us to show that the physical characteristics of the shocks exhibit a strong spatial variation over few atomic scale lengths across 133 sky locations. Our results point to the presence of a population of non-thermal protons (10-100 keV) which might well be the seed particles for generating highenergy cosmic rays. We also present observations of Tycho's supernova remnant taken with the narrow-band tunable filter imager OSIRIS at the GTC and the Fabry-Perot interferometer GHaFaS at the WHT to resolve respectively the broad and narrow $\mathrm{H} \alpha$ lines across a large part of the remnant.
\end{abstract}

Keywords. Balmer emission, supernova remnants, cosmic rays, integral-field spectroscopy

\section{Introduction}

Supernova remnants are laboratories for studying optical shocks. An optical spectrum dominated by Balmer lines is seen when a fast astrophysical shock enters partly neutral interstellar gas. Balmer dominated shocks (BDSs) are characterized by velocities higher than $200 \mathrm{kms}^{-1}$, presence of two-component $\mathrm{H} \alpha$ line, absence of forbidden lines of lowly ionized metals, and general lack of non-thermal X-ray emission at the location where $\mathrm{H} \alpha$ lines are detected (Heng 2010). BDSs are observed around historical supernova remnants (SNRs) like Tycho, Kepler and SN1006.

The hydrogen lines consist of a narrow $\left(\sim 10 \mathrm{kms}^{-1}\right)$ and a broad $\left(\sim 1000 \mathrm{kms}^{-1}\right)$ component. The narrow component is produced by cold neutrals in the pre-shock ambient interstellar medium (ISM) that are collisionally excited by electrons and protons in the shock. While the broad component is produced by post-shock hot neutrals, created through charge exchange between incoming neutrals and hot protons in the shock. Thus, the two-component $\mathrm{H} \alpha$ lines directly yield the pre-shock and post-shock temperatures of the ISM around the remnant. The Balmer line profiles also contain signatures of shock precursors. Investigating in detail the shape of the $\mathrm{H} \alpha$ line has the potential to provide strong observational constraints on cosmic rays (CRs). In particular, the CRs will heat the cold neutrals in the ISM before they are being ionized by the shock, resulting in a narrow $\mathrm{H} \alpha$ line of which the width is broadened beyond the normal $10-20 \mathrm{kms}^{-1}$ gas dispersion. The CRs can also carry away energy from the protons in the post-shock, so that the broad $\mathrm{H} \alpha$ line has a smaller width than allowed by the fast shock velocity. 
Because the CR precursor is typically spatially unresolved, its additional contribution to the narrow-line $\mathrm{H} \alpha$ emission results in a decreasing broad-to-narrow intensity line ratio. The presence of CRs can also cause the shape of the broad $\mathrm{H} \alpha$ line to deviate from a Gaussian profile (Raymond et al. 2010).

Here we present high-spatial resolution spectro-photometric imaging of the remnants SN 1006 and Tycho, and investigate the $\mathrm{H} \alpha$-line profiles in detail.

\section{Data \& Analysis}

\subsection{VIMOS-IFU Observations of SN 1006}

The long-slits typically used to measure the $\mathrm{H} \alpha$ line have low spatial resolutions resulting in the contribution of multiple shock fronts to the measured $\mathrm{H} \alpha$ line. It is then unclear whether the line shape, including its width, are contaminated by this geometric effect. Optical integral-field unit (IFU) spectrographs are able to trace and distinguish multiple, projected shocks. Since BDSs have not been investigated before with IFUs, our aim in this pilot study was to demonstrate that such observations can be executed and that the scientific yields constitute a marked improvement over previous studies.

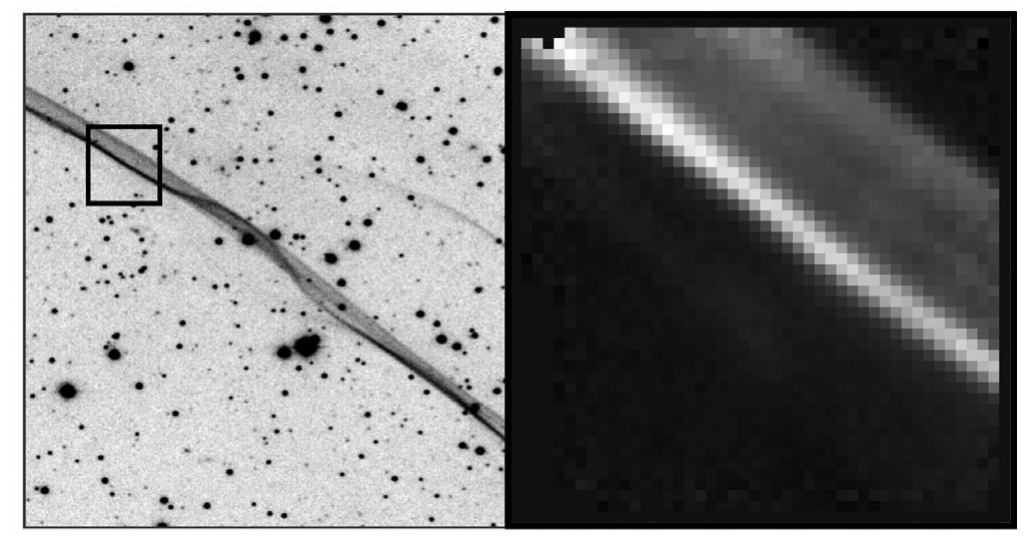

Figure 1. VIMOS-IFU spectroscopy of the shock front in the remnant of SN1006. The left panel shows a CTIO-Curtis-Schmidt narrow-band $\mathrm{H} \alpha$ image (from Winkler et al. 2003) of the NW rim of the remnant with the box indicating the region observed with the VIMOS-IFU. The right panel shows the reduced data cube collapsed in wavelength around the narrow $\mathrm{H} \alpha$ line, nicely recovering the shock in the image.

We used the VIMOS-IFU spectrograph on the Very Large Telescope (VLT) to investigate the fast optical shocks at the northwestern (NW) rim of the remnant of SN1006 (see Figure 1). The $27^{\prime \prime} \times 27^{\prime \prime}$ field-of-view (FOV) of the VIMOS-IFU on a $8 \mathrm{~m}$ telescope enables us to collect enough photons in a reasonable time to reach the high signal-to-noise ratio $(\mathrm{S} / \mathrm{N})$ required to accurately measure the shape of the $\mathrm{H} \alpha$ line, including deviations from a Gaussian profile. The high spatial resolution of $0 .^{\prime \prime} 67\left(\simeq 2 \times 10^{16} \mathrm{~cm}\right.$ at the distance of $\simeq 2 \mathrm{kpc}$ ), in combination with the two-dimensional coverage of the VIMOSIFU allow us to precisely trace the narrow shock front. The spectral resolution with dispersion of $\backsim 48 \mathrm{kms}^{-1}$ is more than sufficient to measure the width of the broad line of 2000-3000 $\mathrm{kms}^{-1}$, as well as deviations from a Gaussian profile. The width of the narrow line of $\backsim 20 \mathrm{kms}^{-1}$ can not be resolved, but its intensity can be accurately measured.

As a first analysis we combined the spectra from four different regions parallel to the shock front and analyzed the resulting two-component $\mathrm{H} \alpha$ lines shown in Figure 2. 

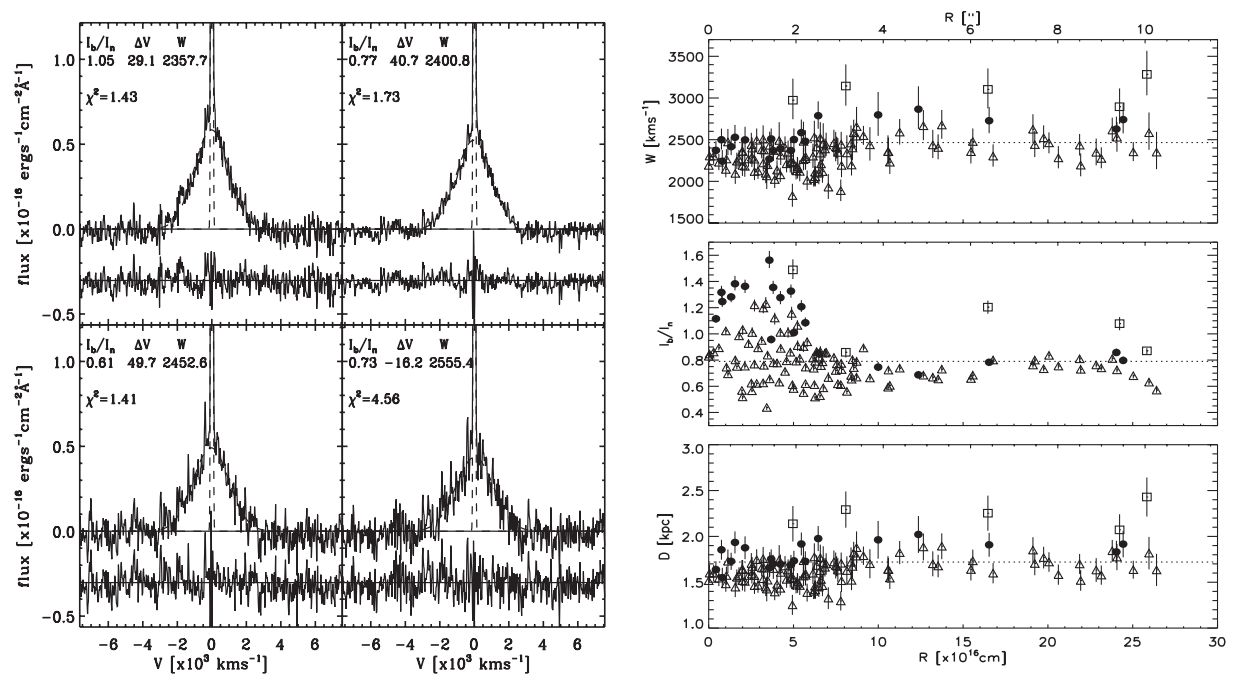

Figure 2. Two-component $\mathrm{H} \alpha$-line profiles and spatial variations of shock parameters. The left four panels show the spectra that have been combined from four different regions parallel to the shock front. In each panel, we show the best-fit parameters: broad-to-narrow intensity ratio $I$, velocity offset $\Delta \mathrm{V}$ from the narrow component (in $\mathrm{kms}^{-1}$ ), and the FWHM of the broad component(in $\mathrm{kms}^{-1}$ ). The three panels on the right show 133 measured (with error bars) broad line widths $W$, broad-to-narrow intensity ratios $I_{b} / I_{n}$, and heliocentric distances $D$ from inferred shock velocities combined with measured proper motions of the shock front (Winkler et al. 2003). Data are ordered in increasing distance from the inner rim to the outer rim. The dashed horizontal lines indicate the measured $W=2465.76 \mathrm{kms}^{-1}$ and $I_{b} / I_{n}=0.79$ values from collapsing all spectra of the pixels on the shock front, and from there the inferred $D=1.72 \mathrm{kpc}$. Data points marked with open triangles (squares) indicate that the $I_{b} / I_{n}$ values are too low (high) to obtain a solution of the van Adelsberg et al. model that does not include non-thermal physics.

Performing double-Gaussian fits we extracted various parameters, including the broadto-narrow line ratio $\left(I_{b} / I_{n}\right)$, velocity offset $(\Delta \mathrm{V})$ from the narrow component and the FHWM of the broad-line component $(W)$. Already strong variation in $I_{b} / I_{n}$ and $W$, as well as hint of non-Gaussianity in the broad-line core point towards the presence of a precursor in the shock.

In order to investigate spatial variations of the physical characteristics across the shock front, we used the method of Cappellari \& Copin (2003) to combine the neighboring pixels and create 133 spatial (Voronoi) bins in which the combined spectra have a minimal S/N of 40. We fitted double-Gaussian to the lines and extracted again the same parameters (per bin) as before: $I_{b} / I_{n}, \Delta \mathrm{V}$ and $W$. We then converted for each bin $W$ and $I_{b} / I_{n}$ to a shock velocity and electron-to-proton temperature ratio $(\beta)$ using the model of van Adelsberg et al. (2008). The variations of the intensity ratios and broad-line widths along with the distances calculated from derived shock velocities and the proper motion measurements of 280 masyr $^{-1}$ (Winkler et al. 2003) are shown in the three right panels of Figure 2. Nearly $85 \%$ of the observed values (represented with empty triangles and squares) are out of the range predicted by the model, which does not include CR physics. Strong spatial variations in the intensity ratios and broad-line widths, the fact that the most of our observed values are not in the model predicted range, and the hint of the non-Gaussianity in the broad-line core all together indicate the potential presence of suprathermal protons, non-thermal particles which might be the seed particles for generating high-energy CRs (Nikolic et al. 2013). 

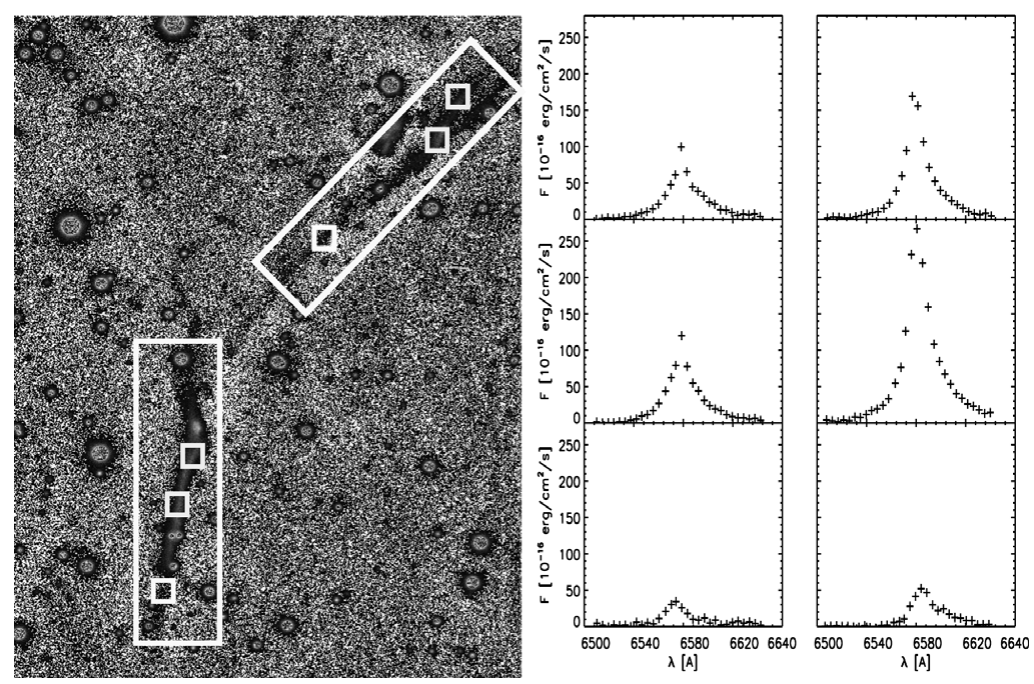

Figure 3. OSIRIS observations of Tycho's remnant. The left panel shows reduced OSIRIS narrow-band imaging data of the northeastern rim of the remnant. Small $5 \times 5$ pixel boxes (white squares) indicate six different positions along the nicely recovered shock front from which the $\mathrm{H} \alpha$ line was extracted and shown in the right panel. The first column shows the spectra from the upper white rectangular region from the top to the bottom, while the second column shows the spectra from the lower white rectangular region again in the same order.

\subsection{OSIRIS \& GHaFaS Observations of Tycho}

Integral-field spectrographs are able to trace and distinguish multiple, projected shocks, but still, the field-of-view of these spectrographs typically covers only a small portion of the full remnant. Henceforth, we use OSIRIS narrow-band tunable filter imaging at GTC (Grand Telescope Canaria). The high spatial resolution of $0 . .^{\prime \prime} 125$ per pixel avoids geometric effects, while the large field-of-view of $4^{\prime} \times 4^{\prime}$ allows to measure the width of the broad component of the $\mathrm{H} \alpha$ line of the shock fronts in the full northeastern part of the remnant of Tycho's supernova. Our goal is to map the changing CR acceleration efficiency along the remnant by measuring broad $\mathrm{H} \alpha$-line widths smaller than expected from the high shock velocities, which we know independently from proper motion measurements of the shock fronts.

OSIRIS low spectral resolution prevents resolving the narrow $\mathrm{H} \alpha$ component. In order to precisely measure the intrinsic narrow $\mathrm{H} \alpha$-line width, we use the Fabry-Perot interferometer GHaFaS at the WHT (William Herschel Telescope) which has the unique capability to scan with a spectral resolution as fine as $8 \mathrm{kms}^{-1}$. This enables us to directly quantify the presence of the $\mathrm{CR}$ precursor. The high spatial resolution of $0 .{ }^{\prime \prime} 2$ plus large FOV 3.' $9 \times 3 .^{\prime} 9$ of GHaFaS allow us to differentiate between different individual shock fronts along Tycho's supernova remnant. Observing exactly the same parts of the Tycho's remnant with both OSIRIS and GHaFaS, we expect to place tight constrains on CR precursors in Tycho's SNR.

\section{Acknowledgments}

We would like to thank Bernd Husemann (AIP), John C. Raymond (HarvardSmithsonian CfA), John P. Hughes (Rutgers University) \& Jesús Falcón-Barroso (IAC) for their collaboration in the work of VIMOS-IFU observations of SN 1006. 


\section{References}

Cappellari, M. \& Copin, Y. 2003, MNRAS, 342, 345

Heng, K. 2010, PASA, 27, 23

Nikolic, S., van de Ven, G., Heng, K., Kupko, D., Husemann, B., Raymond, J. C., Hughes, J. P., \& Falcon-Barroso, J. 2013, Science Express, February 14

Raymond, J. C., Winkler, P. F., Blair, W. P., Lee, J.-J., \& Park, S. 2010, ApJ, 712, 901

van Adelsberg, M., Heng, K., McCray, R., \& Raymond, J. C. 2008, ApJ, 689, 1089

Winkler, P. F., Gupta, G., \& Long, K. S. 2003, ApJ, 585, 324

\section{Discussion}

VINK: Could the non-Gaussianity be caused by a superposition of gaussians with different width along the line of sight? It may be worthwhile to fit the Kappa-profile instead of gaussians.

NiKOLIC: Taking into account the fact that the width of the narrow $\mathrm{H}$-alpha lines (even though unresolved) as well as that shifts between narrow and broad velocity centroids are much smaller than the "extra-core" size of the broad line, the non-Gaussianity can not be due to binning effect, i.e. superposition of Gaussians with different width along the line of sight. We didn't try fitting Kappa-profile because we don't see any physical meaning of it. However, we tried fitting the line with 3 Gaussians as well as fitting Gauss-Hermite polynomial, but none of these have improved the fit. 